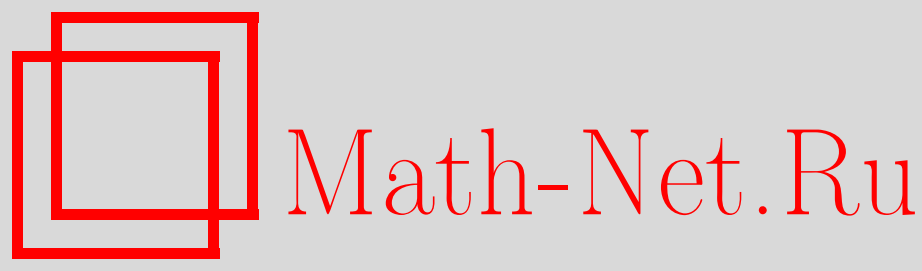

Р. Фиоре, Л. Л. Енковский, В. Либов, М. В. Мачадо, Рождение векторных мезонов в ультрапериферических столкновениях на Большом адронном коллайдере, ТМФ, 2015, том 182, номер 1, 171-181

DOI: https://doi.org/10.4213/tmf8740

Использование Общероссийского математического портала Math-Net.Ru подразумевает, что вы прочитали и согласны с пользовательским соглашением http://www.mathnet.ru/rus/agreement

Параметры загрузки:

IP : 52.6 .47 .48

26 апреля 2023 г., 15:02:07

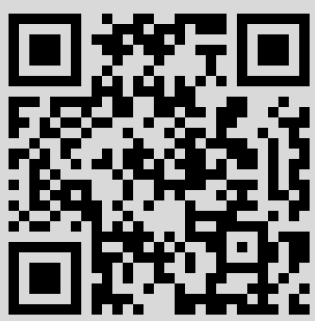




\title{
РОЖДЕНИЕ ВЕКТОРНЫХ МЕЗОНОВ В УЛЬТРАПЕРИФЕРИЧЕСКИХ СТОЛКНОВЕНИЯХ НА БОЛЬШОМ АДРОННОМ КОЛЛАЙДЕРЕ
}

\begin{abstract}
Связь между сечениями реакций с участием фотонов на HERA и ультрапериферическими столкновениями на Большом адронном коллайдере исследована с помощью модели полюсов Редже для рождения векторных мезонов, хорошо описывающей экспериментальные данные ускорителя HERA. Получены предсказания для будущих экспериментов по рождению $J / \psi$ и других векторных мезонов.
\end{abstract}

Ключевые слова: векторные мезоны, коллайдер, HERA, Большой адронный коллайдер, померон.

DOI: $10.4213 / \operatorname{tmf} 8740$

\section{1. ВВЕДЕНИЕ}

После закрытия коллайдера HERA в DESY (Германия) исследование эксклюзивного дифракционного рождения мезонов в ультрапериферических столкновениях протонов и ядер стала одной из приоритетных программ исследований на Большом адронном коллайдере (БАК) [1], [2], что вызвало поток теоретических исследований на эту тему [3]-[7] (обзор можно найти, например, в [8]). Первые экспериментальные результаты о рождении векторных мезонов уже опубликованы [1], [2].

В настоящем исследовании рождения векторных мезонов на БАК мы отмечаем изменение энергетической зависимости сечений при переходе от данных коллайдера HERA к данным БАК. В частности, нас интересует переход от мягких столкновений легких векторных мезонов к тяжелым $\phi, J / \psi, \Upsilon$ и другим мезонам.

${ }^{*}$ Dipartimento di Fisica, Universitá della Calabria, Calabria, Italy

${ }^{\dagger}$ Instituto Nazionale di Fisica Nucleare, Gruppo collegato di Cosenza, Cosenza, Italy. E-mail: fiore@fis.unical.it

${ }^{\ddagger}$ Институт теоретической физики им. Н. Н. Боголюбова НАН Украины, Киев, Украина. E-mail: jenk@bitp.kiev.ua

${ }^{\S}$ DESY, Hamburg, Germany. E-mail: vladyslav.libov@desy.de

`HEP Phenomenology Group, CEP, Porto Alegre, RS, Brazil. E-mail: magnus@if.ufrgs.br 


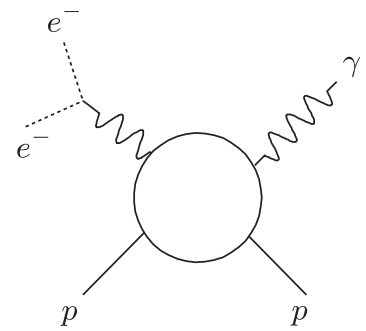

a

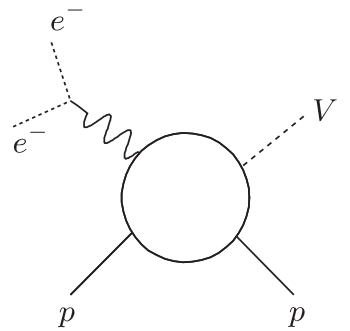

б

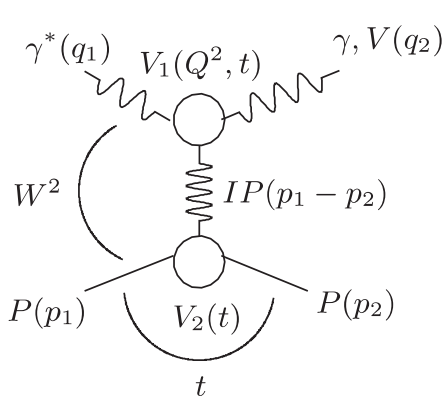

B

Рис. 1. Диаграммы глубоко виртуального комптоновского рассеяния рождения векторных мезонов (а) и (б); амплитуда глубоко виртуального комптоновского рассеяния с факторизацией Редже (в).

\section{2. РОЖДЕНИЕ ВЕКТОРНЫХ МЕЗОНОВ HА HЕRА}

Рождение векторных мезонов на HERA детально исследовалось коллаборациями H1 и ZEUS. Бо́льшая часть событий отбиралась из кинематической области, соответствующей дифракционному рассеянию, вследствие чего процесс может описываться обменом полюса Померанчука - померона (как это показано на рис. 1). Померонный обмен, особенно явный в процессе рождения $J / \Psi$, в котором в соответствии с правилом Цвейга запрещен любой обмен вторичными траекториями, составленными из кварков, кроме померонного обмена. Это означает вовсе не упрощение динамики взаимодействия, а возможность детального изучения с помощью этого класса реакций столь сложного объекта, каким является померон. При этом основной является двойственная проблема померона: он представляется мягким или жестким в зависимости от виртуальности налетающего фотона и/или массы рожденного векторного мезона.

В большинстве работ на данную тему предполагается существование двух померонов: жесткого, или КХД-померона, следующего из пертурбативной квантовой хромодинамики (КХД), а также мягкого, называемого не совсем удачно непертурбативным. Мы, однако, считаем, что в природе существует единый померон, состоящий из двух компонент, относительный вес которых меняется соответствующими весовыми множителями, где мера жесткости есть сумма квадрата виртуальности фотона $Q^{2}$ и массы рожденного векторного мезона $M_{V}^{2}, \widetilde{Q}^{2}=Q^{2}+M_{V}^{2}$.

Конкретная модель, реализующая эту идею, была недавно построена и апробирована сравнением с опытом (см. [9], [10] и ссылки в указанных работах). Соответствующая амплитуда рождения векторных мезонов имеет вид

$$
\begin{aligned}
A\left(s, t, Q^{2}, M_{v}^{2}\right)= & \widetilde{A}_{s} e^{-(i \pi / 2) \alpha_{s}(t)}\left(\frac{s}{s_{0}}\right)^{\alpha_{s}(t)} e^{b_{s} t-n_{s} \ln \left(1+\widetilde{Q}^{2} / \widetilde{Q}_{s}^{2}\right)}+ \\
& +\widetilde{A}_{h} e^{-(i \pi / 2) \alpha_{h}(t)}\left(\frac{s}{s_{0}}\right)^{\alpha_{h}(t)} \times \\
& \times \exp \left[b_{h} t-\left(n_{h}+1\right) \ln \left(1+\frac{\widetilde{Q}^{2}}{\widetilde{Q}_{h}^{2}}\right)+\ln \left(\frac{\widetilde{Q}^{2}}{\widetilde{Q}_{h}^{2}}\right)\right],
\end{aligned}
$$




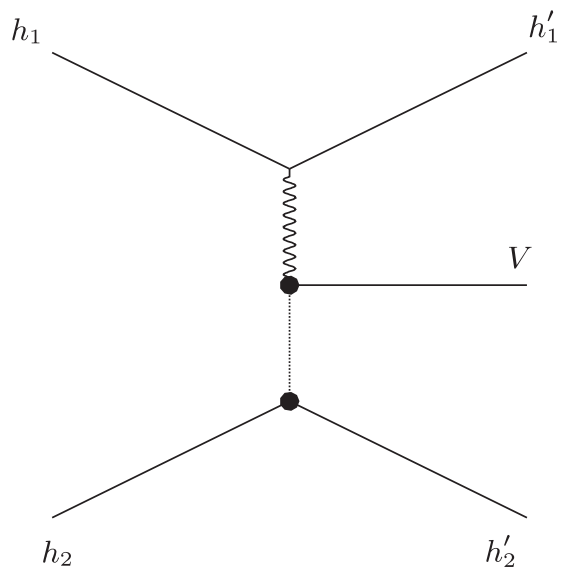

Рис. 2. Диаграмма Фейнмана для рождения векторного мезона при столкновении адронов.

где $\alpha_{s}(t)$ и $\alpha_{h}(t)$ - траектории мягкой и жесткой компонент померона. Напомним, что померон един для всех реакций, меняться могут его компоненты и параметры в них. Соответствующие примеры можно найти в недавних работах [9], [10].

Учитывая экспоненциальный вид дифракционного конуса, интегральное (называемое также полным) сечение рождения векторных мезонов можно легко найти без интегрирования по формуле

$$
\sigma_{\mathrm{el}}(s)=\left.\frac{1}{B(s)} \frac{d \sigma}{d t}\right|_{t=0} .
$$

Поскольку нашей главной целью является сравнение энергетических зависимостей рождения векторных мезонов, полученных на HERA и на БАК, мы начнем с простых выражений для сечения $\gamma p \rightarrow V p$, отложив использование более сложных выражений (1) до будущих исследований.

\section{3. РОЖДЕНИЕ ВЕКТОРНЫХ МЕЗОНОВ НА БАК}

Сечение рождения векторных мезонов (рис. 2) можно записать в факторизованном виде (см. [4], [8], в частности выражения (1) и (9) в первой из работ [4]). Распределение по быстротам $Y$ рожденных векторных мезонов $V$ в реакции $h_{1}+h_{2} \rightarrow$ $h_{1} V h_{2}$ (где $h$ - нуклон, например протон или ядро $\mathrm{pPb}, \mathrm{PbPb}$ и т.п.) вычисляется согласно стандартной процедуре, основанной на факторизации потока фотонов и сечения рассеяния фотона на протоне (см. ниже).

Вообще говоря, дифференциальное сечение рассеяния $\gamma p$ зависит от трех переменных: полной энергии $W$ системы $\gamma p$, квадрата переданного импульса в протонной вершине $t$, а также $\widetilde{Q}^{2}=Q^{2}+M_{V}^{2}$, где $Q^{2}=-q^{2}-$ виртуальность фотона. Поскольку по определению в ультрапериферических столкновениях $b \gg R_{1}+R_{2}$, где $b-$ прицельный параметр, т. е. кратчайшее расстояние между центрами сталкивающихся частиц (ядер), а $R$ - их радиус, фотоны почти реальны, $Q^{2}=0$, единственной мерой жесткости остается $M_{V}^{2}$. Отметим, что это условие может не выполняться 
для периферических столкновений, где $b \sim R_{1}+R_{2}$, а также если заменить обмен фотона помероном или оддероном.

Будем пользоваться сечением рождения векторных мезонов, проинтегрированным по $t$, или, что эквивалентно, соответствующим дифференциальным сечением, деленным на величину наклона переднего конуса. Начнем с простой параметризации сечения $\sigma_{\gamma p \rightarrow V p}(W)$,

$$
\sigma_{\gamma p \rightarrow V p}(W)=\int_{t_{\min }}^{t_{\mathrm{thr}}} d t \frac{d \sigma}{d t}, \quad t_{\min } \approx-\frac{s}{2}, \quad t_{\mathrm{thr}} \approx 0,
$$

предложенного Доннаки и Ландсхофом [11]: $\sigma(W) \sim W^{\delta}, \delta \approx 0.8$ (более сложные модели [9], [10], [12] будут использованы ниже).

Дифференциальное сечение как функция быстроты имеет вид

$$
\begin{aligned}
\frac{d \sigma_{h_{1} h_{2} \rightarrow h_{1} V h_{2}}}{d Y}=\omega_{+} & \frac{d N_{\gamma / h_{1}}\left(\omega_{+}\right)}{d \omega} \sigma_{\gamma h_{2} \rightarrow V h_{2}}\left(\omega_{+}\right)+ \\
& +\omega_{-} \frac{d N_{\gamma / h_{2}}\left(\omega_{-}\right)}{d \omega} \sigma_{\gamma h_{1} \rightarrow V h_{1}}\left(\omega_{-}\right)
\end{aligned}
$$

где $d N_{\gamma / h}(\omega) / d \omega-$ поток “эквивалентных" фотонов [8],

$$
\frac{d N_{\gamma / h}(\omega)}{d \omega}=\frac{\alpha_{e m}}{2 \pi \omega}\left[1+\left(1-\frac{2 \omega}{\sqrt{s}}\right)^{2}\right]\left(\ln \Omega-\frac{11}{6}+\frac{3}{\Omega}-\frac{3}{2 \Omega^{2}}+\frac{1}{3 \Omega^{3}}\right),
$$

$\sigma_{\gamma p \rightarrow V p}(\omega)$ - полное (проинтегрированное по $t$ ) сечение процесса фоторождения векторного мезона (то же, что на HERA, см. [12]), $\omega$ - энергия фотона, $\omega=W_{\gamma p}^{2} / 2 \sqrt{s}$, $\omega_{\min }=M_{V}^{2} / 4 \gamma_{\mathrm{L}} m_{p}$, где $\gamma_{\mathrm{L}}=\sqrt{s} / 2 m_{p}-$ лоренц-фактор (лоренцевский буст одного пучка). Например, для $p p$-рассеяния при $\sqrt{s}=7$ ТэВ имеем $\gamma_{\mathrm{L}}=3731$. Далее, $\Omega=1+Q_{0}^{2} / Q_{\min }^{2}, Q_{\min }^{2}=\omega / \gamma_{\mathrm{L}}^{2}, Q_{0}^{2}=0.71 \Gamma_{\ni} \mathrm{B}, x=M_{V} e^{(-y)} / \sqrt{s}, Y \sim \ln \left(2 \omega / m_{V}\right)-$ быстрота.

Для определенности зафиксируем следующее: а) сталкивающиеся частицы - протоны; б) рожденный векторный мезон $V-J / \psi$, а энергия столкновения равна $\sqrt{s}=7$ ТэВ; в) константы собраны в $A=\alpha_{\mathrm{em}} / 2 \pi c=Q_{0}^{2} \gamma_{\mathrm{L}}^{2}$ (отметим, что форма распределения по $Y$ чувствительна к значению и знаку константы $c$ ). Знаки $i= \pm$ соответствуют первому или второму члену в $(2), \omega_{ \pm} \sim e^{ \pm Y}$.

3.1. Поправки на вероятность выживания быстротного зазора. На полученные результаты может повлиять учет взаимодействия в начальном и конечном состояниях, называемого также перерассеянием. Вычисление этих поправок далеко не однозначно, причем результат вычислений зависит как от затравки, так и от выбранной процедуры унитаризации. Чем лучше (ближе к реальности) затравка, тем меньше поправки и больше вероятность выживания быстротного зазора. Ввиду неоднозначности этого сложного явления, заслуживающего отдельного изучения, выходящего за рамки данной статьи, здесь мы пользуемся лишь общепринятым рецептом [5], умножая амплитуду рассеяния (сечение) на число (меньшее единицы), зависящее от энергии и, возможно, от других кинематических переменных. Значение этой константы для простоты положим равным 0.8 . 


\section{4. РЕЗУЛЬТАТЫ}

Сравним наши теоретические предсказания с экспериментальными данными. При вычислениях мы пользуемся двумя моделями для сечения эксклюзивного фоторождения $\sigma_{\gamma p \rightarrow J / \Psi}-$ моделью со степенным поведением $\sigma_{\gamma p \rightarrow J / \Psi} \sim W^{\delta}$ с $\delta=0.8$ и так называемой геометрической моделью, предложенной и использованной для глубоко виртуального комптоновского рассеяния [11].

Помимо $W$ и $t$ модель содержит также зависимость от виртуальности $Q^{2}$. Параметры подгонялись к данным о глубоко виртуальных комптоновских рассеяниях, полученных на HERA, но модель также может быть с успехом применена к описанию рождения векторных мезонов. Здесь мы будем пользоваться двумя версиями реджеометрической модели [9], [10]. В работе [9] она описывает сечение фоторождения $\left(Q^{2}=0\right)$, а также интегральное сечение (формула (11) работы [9])

$$
\sigma_{\gamma p \rightarrow J / \Psi}=A_{0}^{2} \frac{\left(W / W_{0}\right)^{4\left(\alpha_{0}-1\right)}}{\left(1+\widetilde{Q}^{2} / Q_{0}^{2}\right)^{2 n}\left[4 \alpha^{\prime} \ln \left(W / W_{0}\right)+4\left(a / \widetilde{Q}^{2}+b / 2 m_{N}^{2}\right)\right]},
$$

где $\widetilde{Q}^{2}=Q^{2}+m_{V}^{2}$, а параметры, полученные подгонкой в работе [9] к фоторождению $J / \psi$, приведены в табл. IІ указанной работы: $A_{0}=29.8 \pm 2.8, Q_{0}^{2}=2.1 \pm 0.4 \Gamma_{\ni} \mathrm{B}^{2}$, $n=1.37 \pm 0.14, \alpha_{0}=1.20 \pm 0.02 \Gamma_{\ni} \mathrm{B}^{-2}, \alpha^{\prime}=0.17 \pm 0.05 \Gamma_{\ni} \mathrm{B}^{-2}, a=1.01 \pm 0.11$, $b=0.44 \pm 0.08, W_{0}=1$ ГэВ ${ }^{2}$. Отметим, что, в отличие от исходной формулы, здесь мы заменили $s$ на $W^{2}$, так как $W$ в нашем случае относится к энергии в системе центра масс системы фотон-протон, в то время как $\sqrt{s}-$ энергия в системе центра масс двух протонов.

На рис. За показаны предсказания для распределения по быстротам $Y$ сечения рождения $J / \Psi$ на БАК при $\sqrt{s}=7$ ТэВ. Вообще говоря, степенная и геометрическая модели дают сходные распределения, хотя распределение, которое дает последняя модель, несколько положе. На рис. 3б показана зависимость сечения фоторождения $J / \psi$ от $W$. Здесь предсказания двух моделей близки.

Недавно коллаборацией $\mathrm{LHCb}$ [1], [2] были измерены сечения рождения $J / \Psi$ в зависимости от быстроты. На рис. 4а показано сравнение результатов наших вычислений с этими данными. Как видно из рисунка, экспериментальные точки идут несколько круче обеих теоретических кривых. Из распределений по быстротам коллаборацией $\mathrm{LHCb}$ было получено также сечение фоторождения как функции $W$. Полученный экспериментаторами результат сравнивается с нашими предсказаниями на рис. 4б, где показаны также данные коллабораций ZEUS и H1.

\section{1. Подгонка степенной зависимости к данным коллаборации LHCb.} Как было отмечено выше, в данных коллаборации LHCb распределение по быстротам круче наших предсказаний. Обсудим роль степени $\delta$ в этих распределениях. В частности, выполним подгонку к этим данным с наименьшим квадратичным отклонением. Свободными параметрами являются степень $\delta$ и нормировка. Подгонка дает $\delta=0.37$, а результат показан на рис. 5. Из рисунка видно, что предсказания распределений по быстротам с этим значением параметра оказываются лучше. Поучительно привести сравнение с другими параметризациями, в частности с логарифмической, которая положе степенной (см. рис. 6). Описание с помощью логарифма также хорошее. На рис. 7 показаны предсказания других групп вместе 


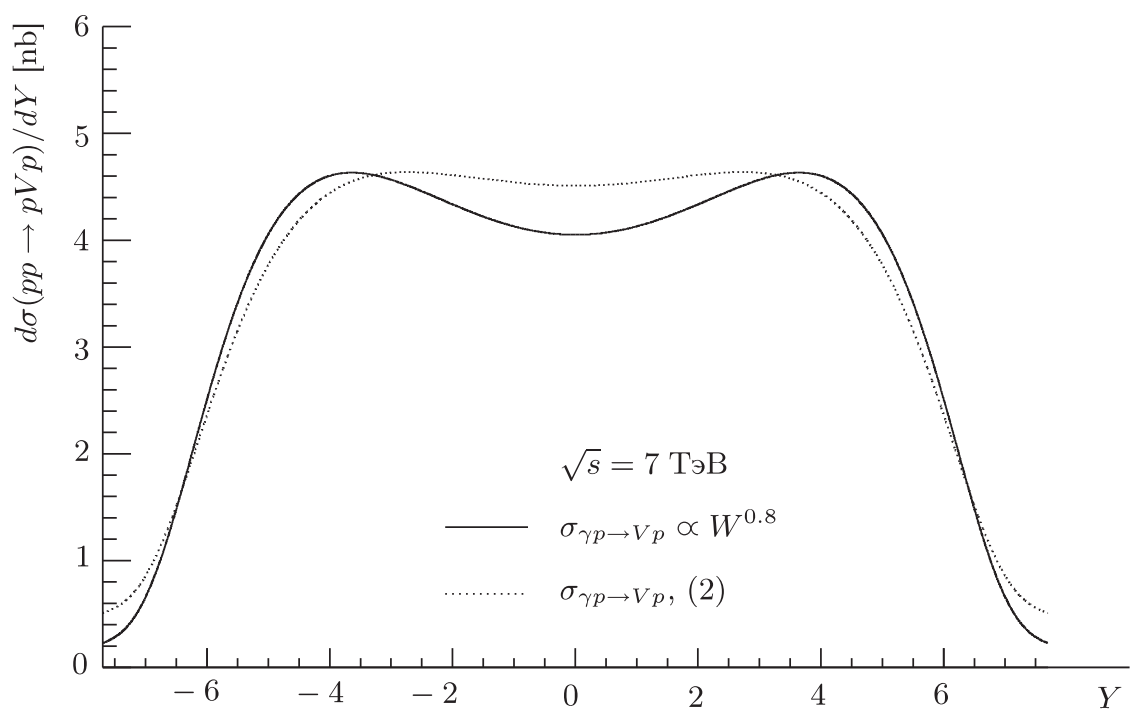

a

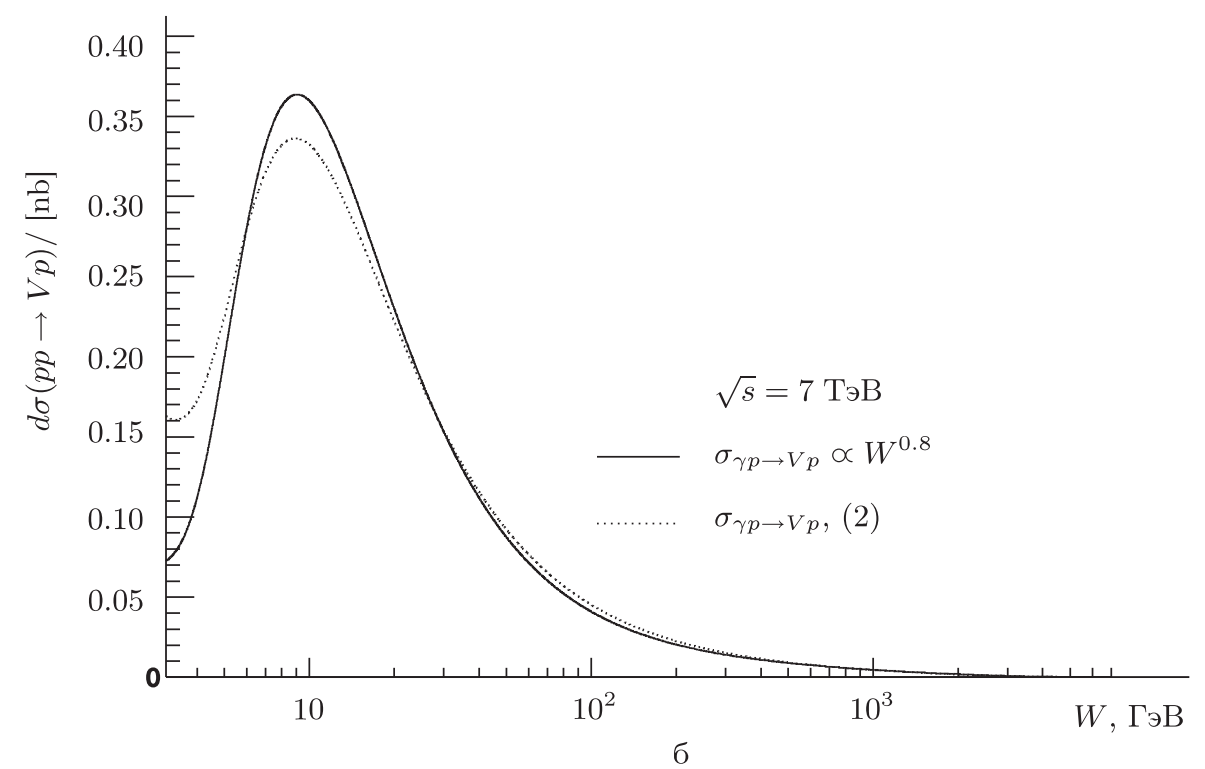

Рис. 3. Дифференциальное сечение эксклюзивного фоторождения $J / \psi$ на БАК как функция быстрот $Y$ (а) и энергии в системе центра масс двух протонов $W$ (б). Сплошные кривые соответствуют простой степенной параметризации сечения рассеяния фотона на протоне, а пунктирные кривые - геометрической модели. 

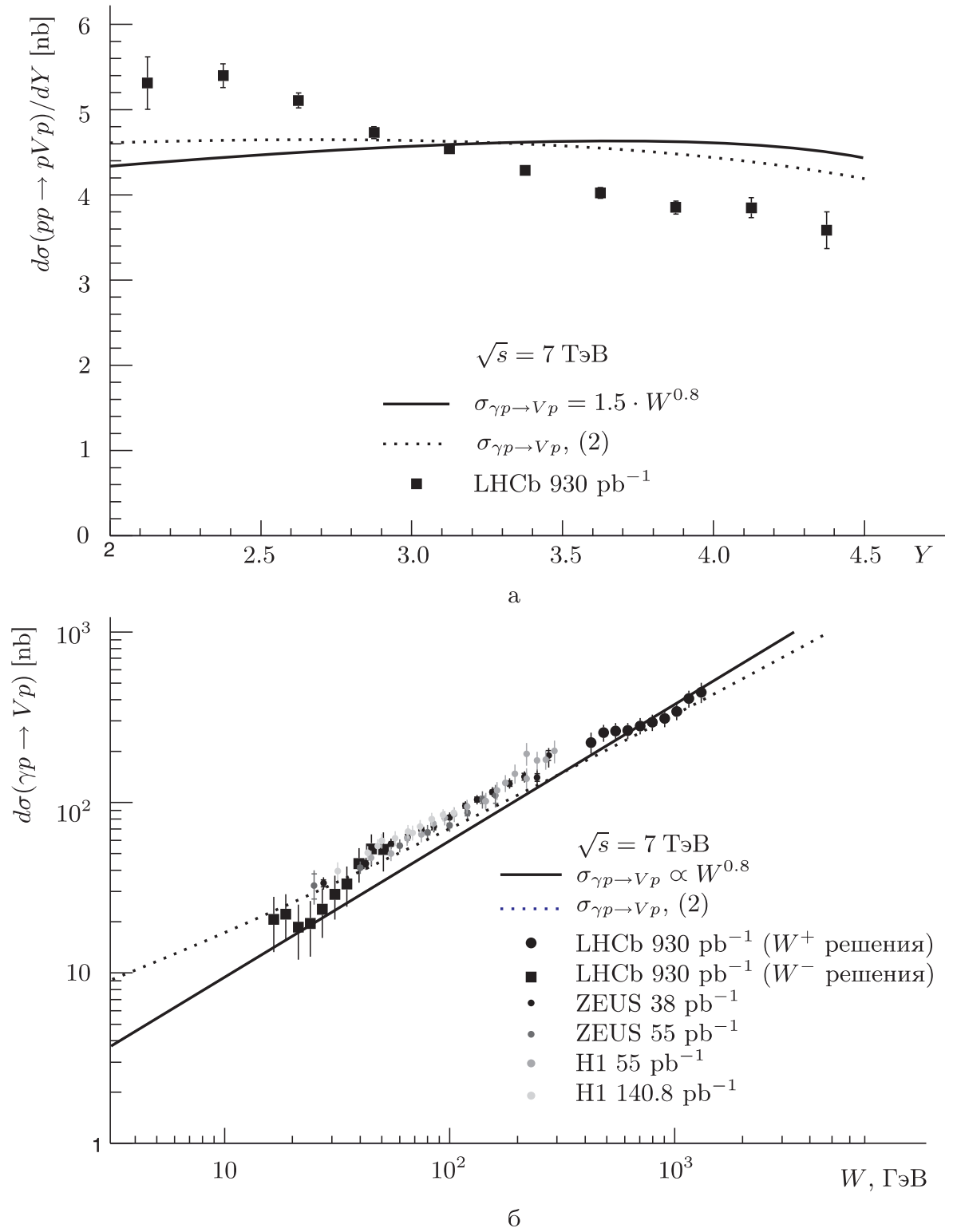

Рис. 4. Дифференциальное сечение рождения $J / \psi$ на БАК как функция быстроты $Y$ и данные LHCb (a). Сечение $\gamma p \rightarrow J / \psi p$ как функция энергии в системе центра масс протонов с данными коллабораций LHCb, ZEUS и Н1 (б).

с распределениями по быстротам данных коллаборации LHCb. Результат описания можно считать разумным.

Наши предсказания сравниваются также с данными коллабораций H1 и ZEUS на рис. 8. Как видно из этого рисунка, лучшее согласие получено при использова- 


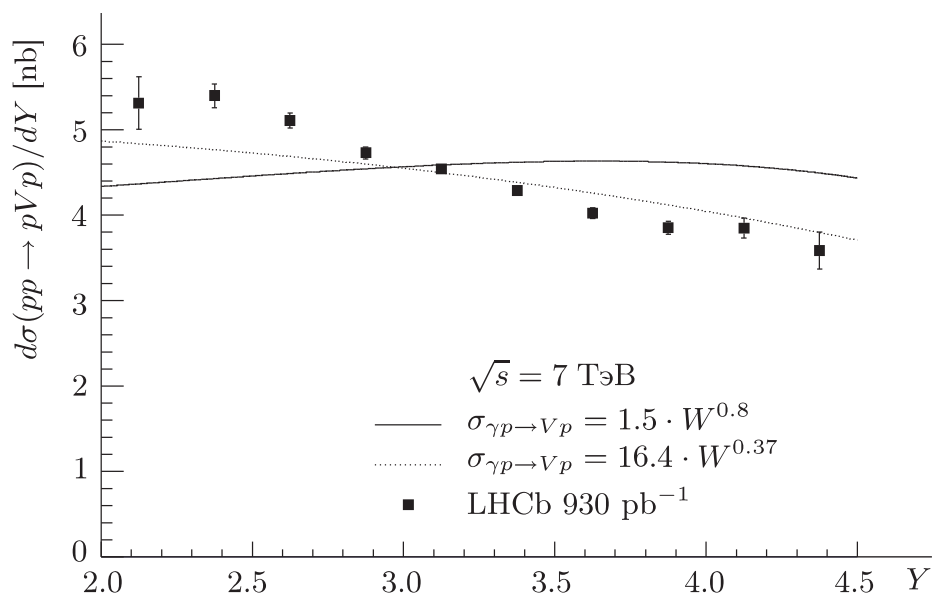

Рис. 5. Дифференциальное сечение рождения $J / \psi$ на БАК как функция быстроты $Y$ и данные LHCb. В вычислениях использована простая степенная параметризация с $\delta=0.8$ (сплошная кривая) и $\delta=0.37$ (пунктирная кривая).

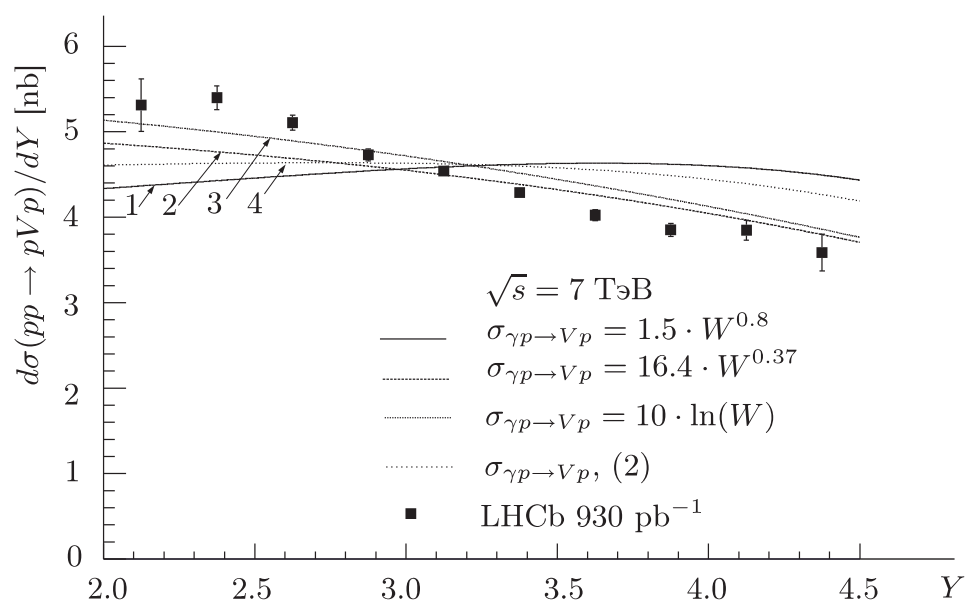

Рис. 6. Дифференциальное сечение рождения $J / \psi$ на БАК как функция быстроты $Y$ и данные LHCb. Для сечения рассеяния фотона на протоне использованы различные параметризации: степенная с $\delta=0.8$ и $\delta=0.37$ (кривые 1 и 2 соответственно), логарифмическая (кривая 3) и геометрическая модель (кривая 4).

нии геометрической модели. Разумное описание дают также степенная параметризация со значением $\delta=0.8$. В то же время при $\delta=0.37$ результаты этой параметризации, как и логарифмической, расходятся с экспериментальными данными. 


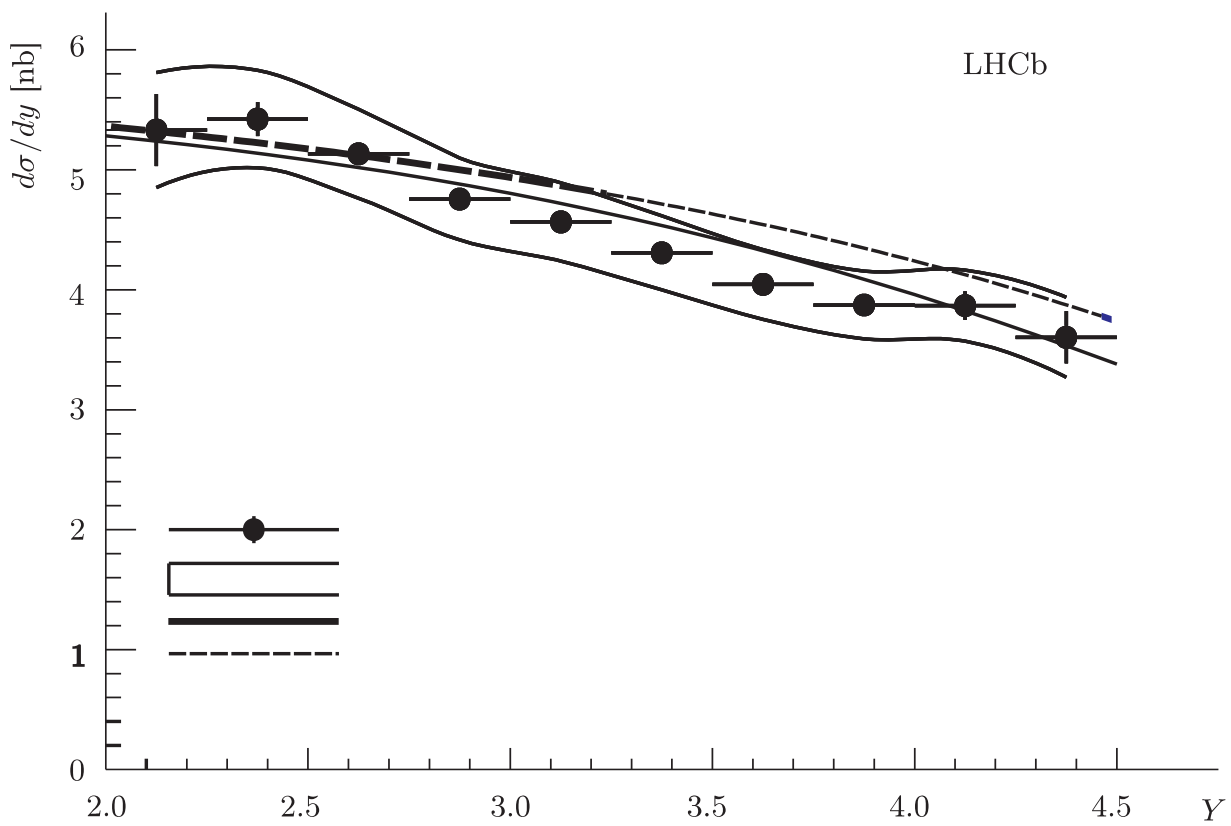

Рис. 7. Дифференциальное сечение рождения $J / \psi$ на БАК как функция быстроты $Y$ и данные $\mathrm{LHCb}[2]$.

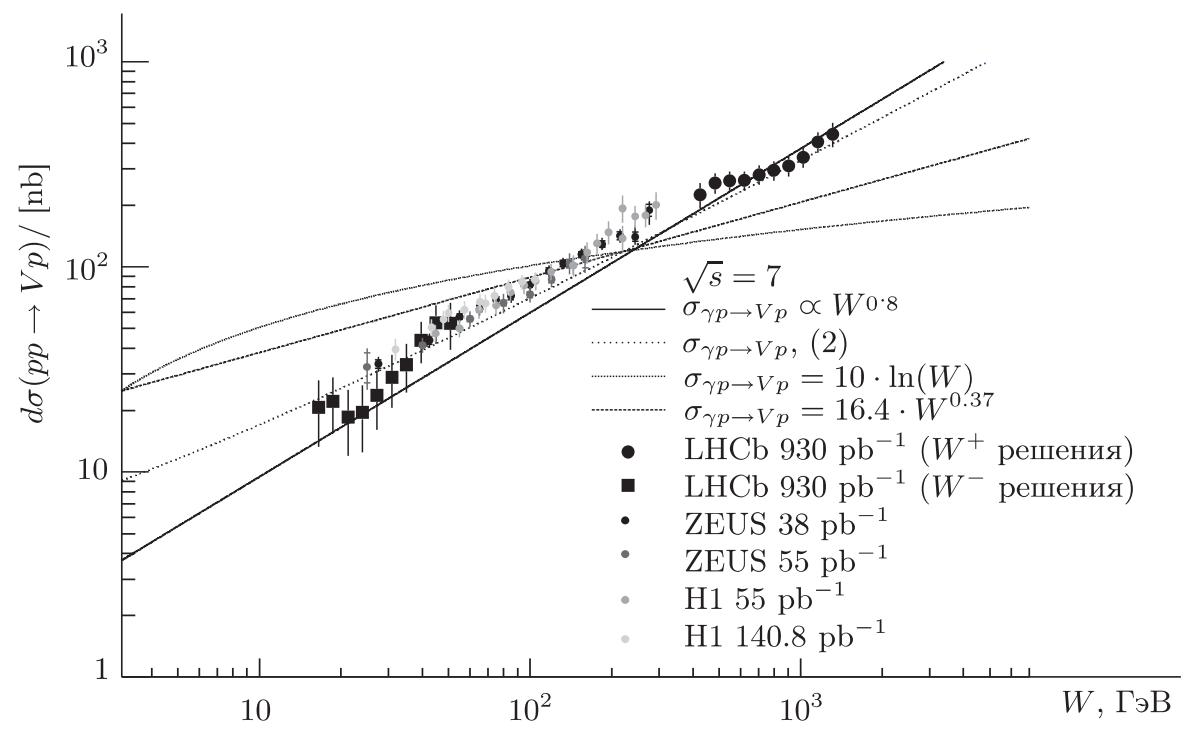

Рис. 8. Сечение фоторождения $(\gamma p \rightarrow J / \psi p)$ в зависимости от энергии в системе центра масс двух протонов в сравнении с данными LHCb, ZEUS и Н1. Обозначения те же, что на рис. 6. 


\section{5. ВЫВОДЫ И ПЕРСПЕКТИВЫ}

В настоящей работе представлены предсказания для эксклюзивного рождения векторного мезона $J / \Psi$ на ускорителе БАК и их сравнение с экспериментальными данными коллаборации LHCb. Для сравнения приведены также данные коллабораций H1 и ZEUS с ускорителя HERA. Для описания фотон-протонных сечений использовались простые степенные параметризации, а также более совершенная геометрическая модель. Быстротные распределения, измеренные LHCb, оказались круче наших предсказаний. Более качественного описания можно достигнуть, подбирая степень роста, что, однако, несовместимо с данными HERA.

Настоящие исследования будут продолжены в следующих направлениях:

1) включение зависимости от $t$, чисто экспоненциальной, а также с отклонениями от нее, соответствующими нелинейным траекториям Редже;

2) учет зависимости сечений $\sigma_{\gamma p \rightarrow V p}$ от $Q^{2}$, пренебрежимой при обмене фотоном, однако существенной при обмене реджеонами (помероном, оддероном и т. п.);

3) изучение неупругих процессов, т. е. таких, в которых рождаются дополнительные частицы за счет излучения глюонов и/или диссоциации протона;

4) более детальное изучение поправок, связанных с вероятностью сохранения быстротного зазора.

Благодарности. Л. Енковский благодарит Физический факультет Университета Калабрии и группу Козенцы Национального института ядерной физики, где была выполнена частично эта работа, за гостеприимство и поддержку. Работа поддержана НАН Украины в рамках гранта "Вещество в экстремальных условиях" и программой DOMUS Венгерской Академии наук.

\section{Список литературы}

[1] R. Aaij, C. Abellan Beteta, A. Adametz et al. [LHCb Collaboration], J. Phys. G: Nucl. Part. Phys., 40:4 (2013), 045001, 18 pp., arXiv: 1301.7084.

[2] R. Aaij, B. Adeva, M. Adinolfi et al. [LHCb Collaboration], J. Phys. G: Nucl. Part. Phys., 41:5 (2014), 055002, 21 pp., arXiv: 1401.3288.

[3] A. Schäfer, L. Mankiewicz, O. Nachtmann, Phys. Lett. B, 272:3-4 (1991), 419-424.

[4] V.P. Gonçalves, M. M. Machado, Heavy quark production in photon-Pomeron interactions at hadronic colliders, arXiv: 1112.3500; Quarkonium $+\gamma$ production in coherent hadron-hadron interactions at LHC energies, arXiv: 1207.5273; Phys. Rev. C, 84:1 (2011), 011902, 4 pp., arXiv: 1106.3036; Phys. Rev. C, 80:5 (2009), 054901, 6 pp., arXiv: 0907.4123; V.P. Gonçalves, W. K. Sauter, Diffractive vector meson production at large-t in coherent hadronic interactions at CERN LHC, arXiv: 1004.1952; V.P. Gonçalves, Probing the Odderon in coherent hadron-hadron interactions at CERN LHC, arXiv: 1211.1207; M. B. Gay Ducati, M. T. Griep, M. V. T. Machado, Phys. Rev. D, 88:1 (2013), 017504, 5 pp., arXiv: 1305.4611; G. Sampaio dos Santos, M. V. T. Machado, Light vector meson photoproduction in hadron-hadron and nucleus-nucleus collisions at the energies available at the CERN Large Hadron Collider, arXiv: 1407.4148.

[5] V. A. Khoze, A. D. Martin, M. G. Ryskin, Eur. Phys. J. C, 24:3 (2002), 459-468, arXiv: hep-ph/0201301; S. P. Jones, A. D. Martin, M. G. Ryskin, T. Teubner, Probes of the small $x$ gluon via exclusive $J / \psi$ and $\Upsilon$ production at HERA and the LHC, arXiv: 1307.7099; Predictions of exclusive $\psi(2 S)$ production at the LHC, arXiv: 1312.6795 .

[6] A. Bzdak, L. Motyka, L. Szymanowski, J.-R. Cudell, Phys. Rev. D, 75:9 (2007), 094023, 12 pp., arXiv: hep-ph/0702134; L. Motyka, G. Watt, Phys. Rev. D, 78:1 (2008), 014023, 13 pp., arXiv: 0805.2113. 
[7] W. Schäfer, A. Szczurek, Phys. Rev. D, 76:9 (2007), 094014, 13 pp., arXiv: 0705.2887; W. Schäfer, G. Slipek, A. Szczurek, Phys. Lett. B, 688:2-3 (2010), 185-191, arXiv: 1003.0610 .

[8] G. Baur, K. Hencken, D. Trautmann, S. Sadovsky, Y. Kharlov, Phys. Rept., 364:5 (2002), 359-450, arXiv: hep-ph/0112211; A. J. Baltz, G. Baur, D. d'Enterria, L. Frankfurt, F. Gelis, V. Guzey, K. Hencken, Yu. Kharlov, M. Klasen, S. R. Klein, V. Nikulin, J. Nystrand, I. A. Pshenichnov, S. Sadovsky, E. Scapparone, J. Seger, M. Strikman, M. Tverskoy, R. Vogt, S. N. White, U. A. Wiedemann, P. Yepes, M. Zhalov, Phys. Rept., 458:1-3 (2008), 1-171, arXiv: 0706.3356.

[9] S. Fazio, R. Fiore, L. Jenkovszky, A. Salii, Acta Phys. Polonica B, 44:6 (2013), 1333-1353, arXiv: 1304.1891.

[10] S. Fazio, R. Fiore, L. Jenkovszky, A. Salii, Phys. Rev. D, 90:1 (2014), 016007, 15 pp., arXiv: 1312.5683 .

[11] A. Donnachie, P. Landshoff, Phys. Lett. B, 296:1-2 (1992), 227-232, arXiv: hep-ph/ 9209205.

[12] M. Capua, S. Fazio, R. Fiore, L. Jenkovszky, F. Paccanoni, Phys. Lett. B, 645:2-3 (1997), 161-166, arXiv: hep-ph/0605319. 